IRRUPCIÓN DE LOS PRECIOS DE TRANSFERENCIA Y LA LUCHA CONTRA EL BEPS

\author{
IRRUPTION OF THE TRANSFER PRICES AND THE FIGHT AGAINST THE BEPS
}

Daniel González Olivares*, Blanca H. Morales Vázquez**, Ariadna Amaro García ${ }^{* * *}$

* M.A. Instituto Tecnológico y de Estudios Superiores de Monterrey, Campus Puebla. Email: danno.gon@gmail.com

** Dra. Benemérita Universidad Autónoma de Puebla.

*** M.A. Benemérita Universidad Autónoma de Puebla. Email: ariadnaamaro@gmail.com

Dirección para recibir correspondencia: blanca.morales.buap@gmail.com

Fecha de recibido: 8 de enero de 2018

Fecha de aceptación: 13 de marzo de 2018

GONZÁLEZ-OLIVARES D., MORALES-VÁZQUEZ B. H., AMARO-GARCÍA A.

MAYO-AGOSTO 2018. AÑO 24, Número 69. Págs. 407-424 
IRRUPCIÓN DE LOS PRECIOS D ETRANSFERENCIA Y LA LUCHA CONTRA EL BEPS

\title{
RESUMEN
}

OBJETIVO: Analizar el concepto, características, contexto y desarrollo de los precios de transferencia así como el impacto del Plan de Acción en contra del BEPS.

MATERIAL Y MÉTODO: De tipo cualitativo, descriptivo y analítico para comprender y analizar los datos. La pregunta de investigación es: ¿Por qué los precios de transferencia son un problema que aqueja a países y empresas de todo el mundo, si ya se han tomado acciones al respecto para realizar un comercio justo para las partes relacionadas?

RESULTADOS: El reto para México es precisar una política económica-fiscal más clara y definida para la inversión extranjera, ya que el escenario no deseable es que su base fiscal se vea afectada por los precios de transferencia, además que se valoren y consoliden los métodos tradicionales de transacción como la forma preferente de ejecutar el principio de plena competencia.

CONCLUSIONES: Se reforzaron los instrumentos y requerimientos para garantizar los beneficios del plan BEPS para actuar en un plano de igualdad y en un marco de trabajo inclusivo con todos los países interesados, con el propósito de implementarlo en la mayor parte del mundo para unificar o estandarizar dichas medidas, así como controlar su impacto en el tiempo.

PALABRAS CLAVE: Globalización. Precios de transferencia. Principio de plena competencia. Vinculación económica. Plan de acción para BEPS.

\begin{abstract}
OBJECTIVE: To analyze the concept, characteristics, context and development of transfer prices as well as the impact of the Action Plan against BEPS (Base Erosion and Profit Shifting).

MATERIAL AND METHOD: Qualitative, descriptive and analytical in order to understand and analyze the data. The research question is: "Why are transfer prices a problem which afflicts countries and companies around the world, if they have already taken action to do fair trade for related parties?"
\end{abstract}




\section{IRRUPCIÓN DE LOS PRECIOS D ETRANSFERENCIA Y LA LUCHA CONTRA EL BEPS}

RESULTS: The challenge for Mexico is to specify a clearer and more defined economic-fiscal policy for foreign investment, since the undesirable scenario is that its tax base is affected by the transfer prices, another challenge is that the traditional methods of transactions are valued and consolidated as the preferred way to execute the arm's length principle.

CONCLUSIONS: The instruments and requirements are reinforced to guarantee the benefits of the BEPS plan to act on an equal basis and in an inclusive framework of work with all the interested countries, aiming to implement it in most of the world to unify or standardize these measures, as well as controlling their impact over time.

KEY WORDS: Globalization. Transfer prices. Arm's length principle. Economic linkage. Action plan for BEPS.

\section{INTRODUCCIÓN}

La globalización entendida como un proceso que ha trastocado economías, tecnologías, políticas, sociedades, empresas y hasta la cultura a escala mundial, ha conllevado a una progresiva comunicación e interdependencia entre los distintos países del mundo uniendo sus mercados, sociedades y culturas, a través de una serie de transformaciones sociales, económicas y políticas que les dan el carácter de global. Este proceso de globalización económica no se podría entender sin la apertura comercial, las innovaciones tecnológicas y sobre todo sin la liberalización de las regulaciones nacionales sobre inversión extranjera para interconectar lo local a lo global y lo global a lo local.

Por lo anterior, la globalización caracterizada por la creciente preponderancia del comercio internacional en el engranaje económico global conllevó a unir a empresas de diferentes países incorporando dinámicas que tienen como centro diferentes espacios, con lo cual las haciendas públicas de los países han tenido que contraer nuevos retos, ya que naturalmente han estado bajo órbitas fiscales diferentes $\mathrm{y}$, durante los últimos años, el papel de las empresas multinacionales en el comercio mundial se ha incrementado grandilocuentemente.

Ahora bien, la expansión de las empresas multinacionales ha implicado el reestructurar sus actividades y operaciones de negocio, esto como una respuesta a los complejos planteamiento GONZÁLEZ-OLIVARES D., MORALES-VÁZQUEZ B. H., AMARO-GARCÍA A. 
IRRUPCIÓN DE LOS PRECIOS D ETRANSFERENCIA Y LA LUCHA CONTRA EL BEPS

fiscales, pero esta aseveración no solo es aplicativo para las empresas sino también para las administraciones tributarias, ya que las normas de tributación de cada país no se pueden considerar aisladamente sino por el contrario, deben ser abordadas en un contexto internacional amplio, con lo cual éstas se han visto obligadas a desempeñar un papel fundamental en la expansión de la integración económica internacional, sin embargo, el mayor reto ha sido a la hora de determinar los ingresos y los gastos de una empresa porque al momento de ser tenidos en cuenta en una jurisdicción han tenido que enfrentarse a la dificultad práctica de cómo hacerlo y es en esta parte donde los precios de transferencia juegan un papel importante dentro de la materia impositiva a determinar.

\section{Definiendo los precios de transferencia}

El concepto de precio de transferencia es dudoso, ya que dependiendo del enfoque multidisciplinario adoptado es como se confiere un significado diferente, ya que si se analizará por parte de la autoridad fiscal representaría a la actividad de fiscalización o si fuera de parte de la empresa implicaría una oportunidad de negocios para no descapitalizarse. En un punto más neutral, por ejemplo la Comisión Económica para América Latina y el Caribe los conceptualiza así:

Así, encontraremos que la disciplina económica se aboca al problema del precio de transferencia de bienes y servicios entre divisiones de una misma empresa. En tanto, las autoridades fiscales de los países se interesan por los precios de transferencias entre compañías relacionadas pertenecientes a grupos multinacionales, en tanto movilicen ganancias desde jurisdicciones con alta carga impositiva hacia otras de baja. Desde una perspectiva económica, los precios de transferencia son instrumentos para lograr la eficiencia económica (Hantke, 2011).

De acuerdo con la Organización para la Cooperación y el Desarrollo Económicos (OCDE), los precios de transferencia son los costos a los que una empresa transfiere bienes físicos e intangibles o presta servicios a empresas asociadas.

En México, el término precios de transferencia se establece en la Ley del Impuesto sobre la Renta (ISR) el cual indica que es el precio pactado en las operaciones comerciales entre dos partes relacionadas, entendiendo por ello la relación entre personas físicas o morales, cuando una de ellas participa directa o indirectamente en la administración, el control o el capital de la otra; o 
IRRUPCIÓN DE LOS PRECIOS D ETRANSFERENCIA Y LA LUCHA CONTRA EL BEPS

bien, cuando una persona o grupo de personas participa directa o indirectamente en la administración, el control o el capital de dichas personas.

Para ser precisos, el primer y segundo párrafo del artículo 179 de la Ley del ISR, expresa lo siguiente:

Artículo 179. Los contribuyentes del Título II de esta Ley, que celebren operaciones con partes relacionadas residentes en el extranjero están obligados, para efectos de esta Ley, a determinar sus ingresos acumulables y deducciones autorizadas, considerando para esas operaciones los precios y montos de contraprestaciones que hubieran utilizado con o entre partes independientes en operaciones comparables. En el caso contrario, las autoridades fiscales podrán determinar los ingresos acumulables y deducciones autorizadas de los contribuyentes, mediante la determinación del precio o monto de la contraprestación en operaciones celebradas entre partes relacionadas, considerando para esas operaciones los precios y montos de contraprestaciones que hubieran utilizado partes independientes en operaciones comparables, ya sea que éstas sean con personas morales, residentes en el país o en el extranjero, personas físicas y establecimientos permanentes en el país de residentes en el extranjero, así como en el caso de las actividades realizadas a través de fideicomisos (Congreso de la Unión, 2018).

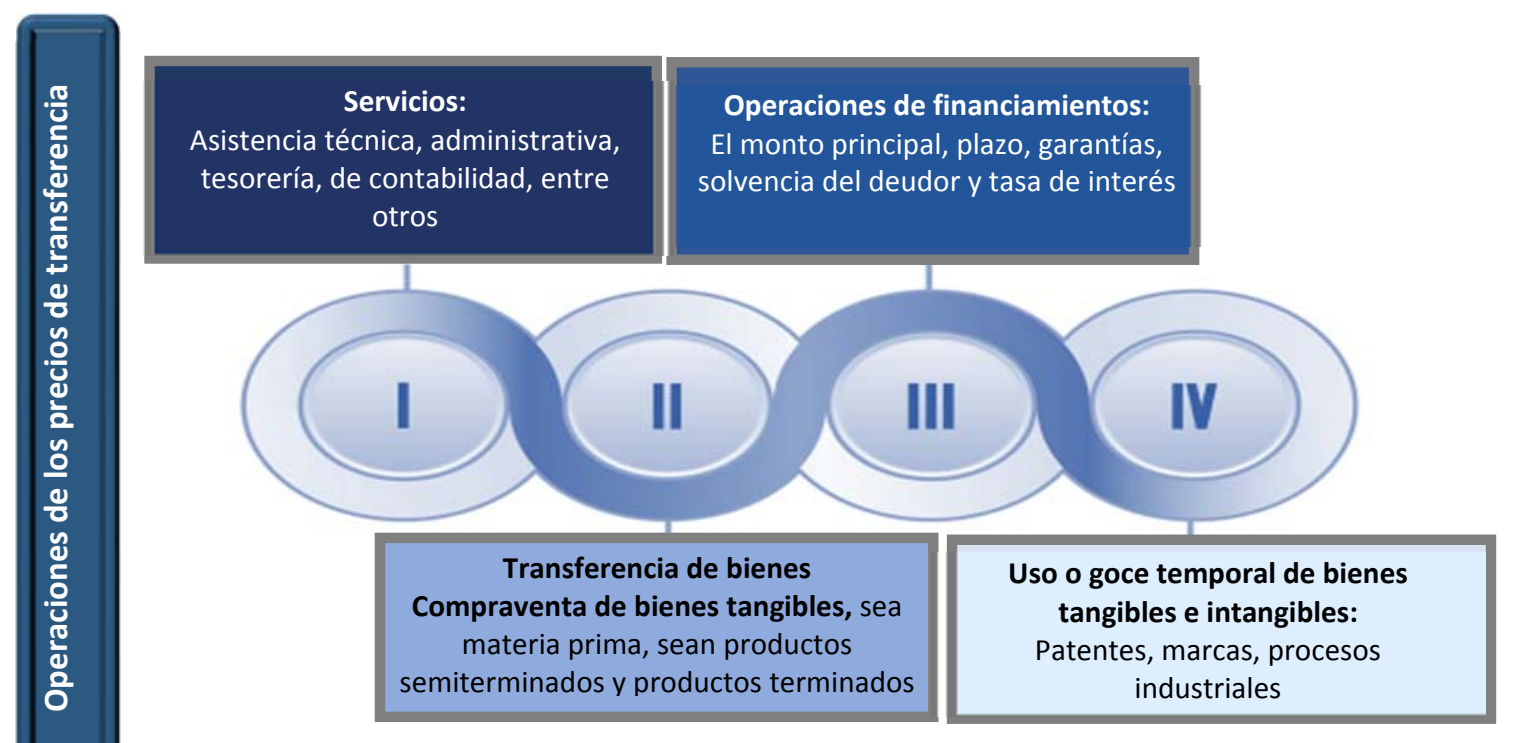

Figura 1. Operaciones en los precios de transferencia de la actividad de las partes relacionadas.

Fuente: Elaboración propia.

GONZÁLEZ-OLIVARES D., MORALES-VÁZQUEZ B. H., AMARO-GARCÍA A.

MAYO-AGOSTO 2018. AÑO 24, Número 69. Págs. 407-424 


\section{IRRUPCIÓN DE LOS PRECIOS D ETRANSFERENCIA Y LA LUCHA CONTRA EL BEPS}

\section{La irrupción de los Precios de Transferencia}

La irrupción de los precios de transferencia se dio consecutivamente en varios países y, por tanto, es complicado señalar con precisión la cronología de cómo determinar su origen, sin embargo, recopilando datos de la OCDE (2018) y de la Comisión de Precios de Transferencia (2011) del IMCP, se señalan de manera muy general los antecedentes de los precios de transferencia.

El origen. Los precios de transferencia tienen su origen conjuntamente con el desarrollo de las grandes corporaciones internacionales que tienen sus matrices en otros países y que ejercen influencia sobre la economía interna de los países que las albergan, en los mercados mundiales de capital y en el comercio, esto es, las llamadas empresas multinacionales. En la medida en que éstas empezaron a desarrollar sus estrategias para aumentar sus beneficios y minimizar sus riesgos por medio de la diversificación de sus actividades productivas, comerciales y financieras, fueron surgiendo las técnicas para manejar los precios de transferencia entre las distintas empresas que conformaban un mismo grupo multinacional.

1915, Reino Unido. Primera jurisdicción fiscal que promulgó una norma interna para regular los precios de transferencia.

1917, Estados Unidos. Emite la reglamentación que establecía la exigencia de declaraciones consolidadas a las empresas asociadas en el Impuesto Federal sobre la Renta de Sociedades, sin embargo fue hasta el año de 1935, que aparece en este país lo que es la norma actual para el tema de precios de transferencia.

1920, OCDE. El Comité Fiscal en colaboración con el Comité Financiero de la OCDE, el 27 de octubre de 1920 iniciaron con el análisis del fenómeno y consecuencias de la doble imposición.

1932, Argentina. Fue el primer país de Latinoamérica que reconoció en su marco jurídico fiscal el tema sobre precios de transferencia.

1979, OCDE. El Comité de Asuntos Fiscales dedicado al estudio continuo de la doble imposición, publicó un reporte denominado "Precios de Transferencia y Empresas Multinacionales", que desarrolló el Principio de Plena Competencia (Arm's Length Principle) como aparece en el artículo 9 del Modelo de Convenio Fiscal sobre la Renta y sobre el Patrimonio.

1994. México. En la legislación mexicana un antecedente importante sobre los Precios de Transferencia surge en el año 1994, con la incorporación de México a la OCDE, ya que de manera GONZÁLEZ-OLIVARES D., MORALES-VÁZQUEZ B. H., AMARO-GARCÍA A. 


\section{IRRUPCIÓN DE LOS PRECIOS D ETRANSFERENCIA Y LA LUCHA CONTRA EL BEPS}

formal México reconoce las recomendaciones y se compromete a aplicar los principios que esta organización emite y así estar en condiciones de cumplir con los objetivos que se tienen, entre ellos, el contribuir a la expansión del comercio internacional en una base multilateral, no-discriminatoria de acuerdo con las obligaciones internacionales. Además, en este mismo año México firma el Tratado de Libre Comercio de América del Norte (TLCAN), con lo cual trae aparejado la inclusión a la globalización y con ello nuevas reglas para el comercio internacional con políticas y finanzas apegadas a derecho. Sin embargo, es hasta el ejercicio fiscal 2015 cuando los requisitos de documentación de precios de transferencia se encontraban contenidos solamente en el artículo 76, fracción IX, de la Ley del Impuesto Sobre la Renta.

1995, OCDE. El Comité de Asuntos Fiscal de la OCDE publicó el 13 de julio las "Guías de Precios de Transferencia para Empresas Multinacionales y Administraciones Fiscales" (Guías de la OCDE). Estas guías son el parteaguas de los primeros esfuerzos por regularizar los precios de transferencia, ya que permitieron de una manera más adecuada seleccionar, aplicar los métodos y desarrollar el análisis de comparabilidad. Cabe señalar que la guía inicio en 1995 con cinco capítulos y concluyó en julio de 2010 con diez capítulos y siete anexos, reestructurando en ese periodo substancialmente los primeros capítulos.

1996 y 2002 Otros países latinoamericanos. Resulta que entre el primer registro de país Latinoamericano que incorporó en su marco jurídico fiscal sobre precios de transferencia (Argentina 1932) y otros países latinos hubo una demora significativa para que se promulgara una regulación sobre la materia al respecto. Países como Brasil y Colombia tuvieron su antecedente en 1996 y 2002, respectivamente, por mencionar alguno de ellos.

Actualidad. A pesar de los grandes esfuerzos que se han hecho por parte de las empresas, los países y sus gobiernos e inclusive organizaciones como la OCDE, la cual ha influido de manera sustancial en el tema, las guías emitidas por esta organización no dejan de ser meras recomendaciones (ya que cada país debe de validarlas mediante sus vías legislativas correspondientes), los precios de transparencia siguen siendo una problemática mundial, ya que siguen siendo una constante de acuerdos que, en algunos casos se favorecen más unos que otros. Cabe señalar que para el caso particular de México, la legislación sobre precios de transferencia se ha hecho apegada a los lineamientos emitidos por la OCDE; sin embargo, otros países, por ejemplo Estados Unidos de América, ha tomado como referencia éstos y ha tropicalizado o domesticado su legislación atendiendo a sus propias necesidades respecto al tema. 


\section{IRRUPCIÓN DE LOS PRECIOS D ETRANSFERENCIA Y LA LUCHA CONTRA EL BEPS}

\section{Precios de transferencia, una problemática de nivel mundial}

Si comenzamos con la pregunta del ¿por qué los precios de transferencia son un problema a nivel mundial, aun a pesar de que se han tenido avances significativos en el tema?, la respuesta sería simple y esto es porque en la parte práctica, cuando los precios de transferencia utilizados no concuerdan con las normas internacionales aplicables o con el principio de plena competencia establecido en el derecho interno pueden distorsionar la distribución del beneficio entre los países en los que opera una empresa multinacional, ya que finalmente se le está negando la posibilidad de percibir ingresos tributarios esenciales, inclusive este desvío puede ser considerado como evasión fiscal, lo cual quebranta la legitimidad y la credibilidad del sistema tributario en su conjunto, desalentando así el cumplimiento de las obligaciones tributarias por parte de todos los contribuyentes. Se trata de cuestiones que afectan a países en desarrollo y desarrollados por igual.

En las Directrices de la OCDE sobre precios de transferencia, se precisa que los problemas en materia de impuestos se dan porque las normas nacionales separadas para la imposición de las empresas multinacionales no pueden considerarse aisladamente, sino que deben abordarse en un contexto internacional amplio:

Los precios de transferencia siguen siendo una prioridad en la agenda de las administraciones tributarias y de los contribuyentes por igual. Los gobiernos deben garantizar que los beneficios imponibles de las multinacionales no sean desplazados artificialmente fuera de su jurisdicción y que la base impositiva comunicada por las empresas multinacionales en su país refleje la actividad económica que se lleva a cabo y los contribuyentes necesitan una orientación clara sobre la aplicación correcta del principio de plena competencia (Martínez, 2017).

\section{Principio de plena competencia}

Muchos países han minimizado el problema de los precios de transferencia abordando los riesgos tributarios que genera su determinación adoptando normativas tributarias basadas en el "principio de plena competencia”. La mayoría de los convenios sobre doble imposición también incluyen el principio de plena competencia como base para la distribución de beneficios y, por consiguiente, de impuestos entre empresas asociadas. 
IRRUPCIÓN DE LOS PRECIOS D ETRANSFERENCIA Y LA LUCHA CONTRA EL BEPS

Dicho lo anterior, es necesario entonces explorar en que consiste el principio de la plena competencia o también conocido en la terminología anglosajona como «Arm's Length» (OCDE, 2017), principio aceptado internacionalmente, el cual establece la política de precios para las empresas con partes relacionadas, con la característica de que sean comparables con los de las operaciones celebradas entre partes no relacionadas y así poder definir el precio o ajustar las diferencias que afectan al mismo.

Según la OCDE, en el principio de la plena competencia, el cual es una norma internacional que debe ser utilizada para la determinación de los precios de transferencia con fines fiscales, tal como lo han acordado sus países miembros. Dicha norma se expone en el apartado 2, del Modelo de Convenio Tributaria sobre la Renta y el Patrimonio de la OCDE (2010) a saber:

Durante la segunda fase se fija un precio a toda transacción efectuada con empresas asociadas atribuida al establecimiento permanente, siguiendo las pautas contenidas en las Directrices de la OCDE sobre Precios de Transferencia, y estas Directrices se aplican por analogía a las operaciones entre el establecimiento permanente y las restantes partes de la empresa de la que forma parte. Este proceso implica la fijación de un precio de plena competencia para las operaciones internas consideradas como tales, mediante:

- la determinación de la comparabilidad entre las operaciones internas y las transacciones en el mercado libre, aplicando directamente los factores de comparabilidad contenidos en las Directrices (características de la propiedad o de los servicios, condiciones económicas y estrategias empresariales) o por analogía (análisis funcional, condiciones contractuales) a la luz de las circunstancias concretas del establecimiento permanente; y - la aplicación por analogía de uno de los métodos de las Directrices a fin de determinar la contraprestación de plena competencia de las operaciones internas entre el establecimiento permanente y el resto de la empresa, teniendo en cuenta las funciones desarrolladas por el establecimiento permanente y los activos y riesgos que se le han atribuido, a este y a otras partes de la empresa.

Este principio pretende evitar prácticas de especulación que resulten en beneficios fiscales para las empresas; además, de que permite supervisar que las operaciones realizadas entre dichas empresas se realicen teniendo como base los precios que se establecen en el mercado por la oferta y la demanda.

En consonancia con lo expuesto se tiene entonces que el contribuyente es responsable de conservar en su contabilidad la documentación que compruebe que se utilizó para determinar las GONZÁLEZ-OLIVARES D., MORALES-VÁZQUEZ B. H., AMARO-GARCÍA A. 
IRRUPCIÓN DE LOS PRECIOS D ETRANSFERENCIA Y LA LUCHA CONTRA EL BEPS

partidas de los ingresos, compras y gastos referentes a las transacciones celebradas con partes relacionadas residentes en el extranjero, para demostrar que las operaciones realmente fueron comparables a valor de mercado efectuadas de acuerdo a los precios o montos de contraprestaciones que se hubieran celebrado con terceros. Además de lo descrito, hay que señalar que antes de determinar el precio de transferencia se deberá realizar un análisis que ubique la operación entre las partes relacionadas y la función que desempeña, el tipo de activo que se administra y los riesgos en que incurre cada contribuyente para observar la congruencia de la transacción con respecto a las actividades que realizan las partes relacionadas puesto que del análisis funcional se determina el tipo de comparables a utilizar y el método para establecer el precio de transferencia, concluyendo que a mayor función, activos y riesgos, se espera un mayor margen de utilidad.

Si lo anterior no sucediera así, se estaría en el entendido de que si las autoridades fiscales involucradas supieran que las operaciones son efectuadas a precios que no son los que rigen en el mercado, necesariamente una de las partes estaría declarando un mayor o menor ingreso y la otra parte declararía un mayor o menor costo, con la repercusión en cada caso de la base gravable y, por ende, del impuesto a declarar, lo cual desfavorecería consecuentemente a alguna de las partes.

\section{Los métodos para la determinación de los precios de transferencia}

Las guías de la OCDE contemplan dos formas para determinar un precio de transferencia, clasificándose en: a. Métodos tradicionales, lo cuales son directos y confiables con base en el principio de libre competencia, comparando los precios de transacciones controladas con los precios en transacciones no controladas comparables con empresas externas y; b. Métodos alternativos, los cuales analizan las utilidades que se originan de transacciones. Para efectos de México, los métodos que el contribuyente podrá aplicar, se encuentran en el artículo 179 de la Ley del ISR.

Los contribuyentes deberán aplicar en primer término el método de precio comparable no controlado, y sólo podrán utilizar los otros métodos, cuando éste no sea el apropiado para determinar que las operaciones realizadas se encuentran a precios de mercado de acuerdo con las Guías de Precios de Transferencia para las Empresas Multinacionales y las Administraciones Fiscales a que se refiere el último párrafo del artículo 179 de la Ley del ISR, asimismo la prioridad 
IRRUPCIÓN DE LOS PRECIOS D ETRANSFERENCIA Y LA LUCHA CONTRA EL BEPS

sobre los demás modelos será aplicando primero los tradicionales discriminando cada método, continuando con los alternativos, hasta dejar el método que más se ajuste a la transacción.

\section{Plan de acción contra la erosión de la base imponible y el traslado de beneficios}

La Organización para la Cooperación y Desarrollo Económico, OCDE (2013c), a instancia del G20, publicó el "Plan de Acción contra la Erosión de la Base Imponible y el Traslado de Beneficios" en julio de 2013. El Plan de Acción contra la Erosión de la Base Imponible y el Traslado de Beneficios es el resultado de la preocupación de distintos países con el actuar de algunas empresas multinacionales que aprovechan las lagunas en la interacción entre los distintos sistemas tributarios con el fin de disminuir artificialmente las bases imponibles y trasladar los beneficios a países o territorios de baja tributación en los que realizan poca o ninguna actividad económica. Para comprenderlo mejor, actualmente la OCDE (2013a) señala que la erosión de la base imponible y el traslado de beneficios se relacionan sobre todo con ejemplos en los que las diferentes normas impositivas producen: Una doble exención, una imposición inferior a la ordinaria y estructuras artificiosas. Por consiguiente, el Plan de Acción contra la Erosión de la Base Imponible y el Traslado de Beneficios, también llamado "Plan de acción BEPS" por sus siglas en inglés (Base Erosion and Profit Shifting), contiene 15 acciones que pretenden prevenir y evitar estas prácticas en deterioro en la recaudación fiscal a nivel internacional.

A manera de resumen, conforme a lo reportado por la OCDE (2018), se explica en que consiste cada una de las acciones del Plan BEPS:

Acción 1: Abordar los retos fiscales de una economía digital. Contempla normas y mecanismos dirigidos a la recaudación en materia del Impuesto al Valor Agregado en el país en donde se encuentra el consumidor, asimismo genera las estrategias para evitar prácticas elusivas en operaciones con activos intangibles o en la implementación fraudulenta del EP. La OCDE y el G-20 continuarán trabajando en un seguimiento de los avances y revisar y analizar la información que, con el tiempo, vaya haciéndose pública.

Acción 2: Neutralizar los efectos de vehículos híbridos. Formula una serie de recomendaciones que tienen como finalidad el que no se utilicen mecanismos y vehículos híbridos con el ánimo de obtener ventajas, lo cual sucede precisamente por la falta de leyes coherentes o asimétricas en materia fiscal tal es el caso cuando nos encontramos frente a la doble deducción, a la doble exención, a los descuentos y a las deducciones múltiples, razón por la cual esta acción desarrolla una metodología común que facilite la convergencia de las prácticas GONZÁLEZ-OLIVARES D., MORALES-VÁZQUEZ B. H., AMARO-GARCÍA A. 
IRRUPCIÓN DE LOS PRECIOS D ETRANSFERENCIA Y LA LUCHA CONTRA EL BEPS

nacionales, por medio de normas internas y convencionales dirigidas a neutralizar tales mecanismos, en otras palabras, las normas propuestas tienen como objeto alinear el tratamiento fiscal de un instrumento o entidad híbrida con aquel aplicado en la jurisdicción contraparte partiendo de una evaluación detenida al pago que causó la deducción, el cual no se encuentra sujeto a gravamen o se encuentra sujeto a un gravamen reducido en el país donde se recibe el ingreso.

Acción 3: Fortalecer reglas CFC. Enuncia una serie de recomendaciones que contribuirán a diseñar normas más efectivas en las compañías foráneas controladas, sin que cada jurisdicción, en función de sus prioridades, pueda conferir a estas normas objetivos políticos diferentes, ya que la acción dota a los países de cierta flexibilidad en cuanto a la compatibilidad en la aplicación de las normas dentro de su marco tributario, esto es, buscan orientar la determinación del tipo de rentas a incluir en el régimen. En definitiva estas recomendaciones están diseñadas para garantizar que las jurisdicciones que opten por implementarlas sean capaces de impedir de una mejor manera que los contribuyentes trasladen beneficios a filiales no residentes.

Acción 4: Limitar la erosión a través de la deducción de intereses. Formula una serie de recomendaciones que buscan diseñar e implementar normas que eviten la erosión de la base imponible a través de la utilización artificiosa en las deducciones por intereses y, por ende, la deducción excesiva de intereses, ya que este tipo de prácticas de los grupos multinacionales para multiplicar fácilmente el nivel de deuda de una entidad del grupo mediante el financiamiento intragrupo son comunes por la vulnerabilidad de las normas fiscales. De forma más puntual, propone una metodología que incluya en la legislación interna la regla de proporcionalidad fija, como mecanismo para limitar la deducibilidad de intereses disponiendo un porcentaje máximo, a su vez se sugiere regular la deducción a través de la regla de proporcionalidad global, con la finalidad de proporcionar una mayor flexibilidad a los grupos empresariales, con esto se le permitiría a las entidades superar el límite de la proporcionalidad fija en su país y deducir el gasto de intereses, sin que supere la proporción de los intereses pagados a terceros.

Acción 5: Combatir prácticas fiscales utilizando la transparencia y la sustancia. Propone una metodología que permite evaluar si existe una actividad sustancial en un régimen preferencial como resultado de las prácticas perniciosas que se centran en los regímenes preferenciales que pueden utilizarse para el traslado artificial de beneficios y en la falta de transparencia de ciertas confirmaciones de criterio, básicamente la acción tiene como objetivo el de limitar la provisión de incentivos fiscales a contribuyentes que no tienen una actividad real en los países que los otorgan, GONZÁLEZ-OLIVARES D., MORALES-VÁZQUEZ B. H., AMARO-GARCÍA A. 
IRRUPCIÓN DE LOS PRECIOS D ETRANSFERENCIA Y LA LUCHA CONTRA EL BEPS

así como fomentar y mejorar la transparencia de la información, en particular mediante el intercambio obligatorio y espontáneo de tax rulings.

Acción 6: Prevenir el abuso de tratados. Señala un estándar mínimo requerido así como nuevas normas para prevenir el abuso de la búsqueda del convenio más favorable, si es que el propósito principal de una operación o transacción es utilizar sus beneficios. Finalmente la acción busca impedir la concesión de los beneficios de los convenios internacionales para evitar la doble imposición en circunstancias inapropiadas, toda vez que estos no han sido previstos para generar una doble exención, sino para evitar la doble imposición, por lo cual debe existir una coherencia entre la realidad económica de los negocios y la fiscalidad, limitando al derecho de los contribuyentes a configurar sus negocios libremente.

Acción 7: Prevenir la evasión artificial del concepto de establecimiento permanente. Su punto de partida es la necesidad de actualizar la definición de establecimiento permanente para evitar el uso de estrategias comunes con fines elusivos, esto es, los convenios fiscales prevén que los beneficios de una empresa no residente sean gravables en un Estado siempre y cuando ésta tenga un establecimiento permanente en dicho Estado al que se le puedan atribuir beneficios.

\section{Acción 8-10: Asegurar que los resultados de los precios de transferencia están alineados} con la creación de valor. En atención a estas tres acciones, se han dividido en tres áreas que refuerzan y clarifican los estándares sobre los precios de transferencia reforzando el principio de plena competencia y el establecimiento de una metodología para garantizar la determinación de precios adecuados para los intangibles de difícil valoración. A continuación de manera breve se señala en que consiste cada acción (8, 9 y 10):

Acción 8: Se enfoca en las transacciones que involucren activos intangibles en relación al trato fiscal que se le ha de dar, en específico se adopta una definición amplia de qué es un intangible, que los beneficios estén debidamente asignados de conformidad con la creación de valor y a desarrollar normas precisas que regularicen el reparto de costos.

Acción 9: Hace referencia a la atribución artificial contractual de riesgos además de sentar las pautas para los beneficios atribuibles a las entidades que conceden fondos en el ámbito del grupo, en contraste con lo anterior sólo se respetarían los contratos cuando dichas atribuciones se correspondieran efectivamente con la toma de decisiones. 
IRRUPCIÓN DE LOS PRECIOS D ETRANSFERENCIA Y LA LUCHA CONTRA EL BEPS

Acción 10: En particular, esta acción afronta otras áreas de alto riesgo, tales como las transacciones susceptibles a calificarse nuevamente por parte de las autoridades fiscales por carecer de razón de negocio razón por la cual se generan situaciones en las que se produce una aplicación inapropiada de los métodos de valoración, y la neutralización de determinados cargos entre empresa, tal como sucede con los pagos de servicios corporativos, gastos de administración, pagos de intereses, fondeo de capital gastos de gestión u operaciones que involucran materias primas o servicios intercompañías de bajo valor añadido, muy focalizada para países en vías de desarrollo.

Acción 11: Evaluación y seguimiento de BEPS. En esta acción se demuestra que derivado de las diferentes fuentes de información y métodos de cálculo es un hecho que las empresas realizan el traslado de beneficios por razones fiscales, lo cual ya se ha mencionado que no es correcto fiscalmente hablando. Dicho lo anterior, medir el alcance de BEPS es un desafío dado la complejidad del fenómeno, ya que por sus propias limitaciones existentes, la consecuencia real es la gran pérdida recaudatoria global del impuesto sobre sociedades. Actualmente, la OCDE trabaja en establecer parámetros e indicadores mucho más equitativos y para ello, ha elaborado una tabla con seis indicadores de BEPS.

\section{Acción 12: Exigir a los contribuyentes que revelen sus mecanismos de planificación fiscal} agresiva. En esta acción el objetivo es enfrentar la falta de información puntual y completa sobre las estrategias fiscales agresivas por parte de las administraciones tributarias en todo el mundo, entonces como una medida de contribuir a su objetivo, se ha establecido un marco armonizador de guías basadas en mejores prácticas, las cuales han sido diseñadas, principalmente, para aquellos países que no cuenten con normas de declaración obligatoria y que si quieren establecer un régimen que les permita cumplir correctamente con sus obligaciones, las cuales les permiten recibir en tiempo y forma la información sobre las estrategias de planificación fiscal agresiva o abusiva y de sus usuarios. Sin embrago, estas guías no son excluyentes y sólo sirven a países que no cuentan con regímenes de declaración obligatoria, ya que los países que si cuentan con uno al implementarlas, les garantiza una mejor eficacia a sus mecanismos de planeación fiscal, tale es el caso de México que desde 2014 las ha contemplado en las disposiciones contenidas en el artículo 31-A del CFF o en sus numerosas informativas que abordan el tema, con una declaración informativa específica sobre operaciones relevantes, en el formato 76, para lograr este propósito. 


\section{IRRUPCIÓN DE LOS PRECIOS D ETRANSFERENCIA Y LA LUCHA CONTRA EL BEPS}

Acción 13: Reexaminar la documentación sobre precios de transferencia. El objetivo de esta acción es incrementar la calidad de la información proporcionada a las administraciones fiscales, ya que con esto se repercute directamente en la reducción de los costos de cumplimiento para las empresas. Tal como lo señala Alvarado H. y Vera R.F. (2016) la estandarización se realiza en tres niveles de la documentación sobre precios de transferencia se puede categorizar en el: Master file, local file y Country by country report.

Acción 14: Hacer más efectivos los mecanismos de resolución de controversias. El objetivo de esta acción es que los países se comprometan a mejorar la rapidez en la resolución de conflictos para luchar contra los obstáculos que impiden que los países resuelvan las controversias relacionadas con los convenios mediante los procedimientos amistosos y para ello se hace necesario incluir un arbitraje preceptivo y vinculante en sus convenios fiscales bilaterales para evitar provocar cierta inseguridad jurídica y así no caer en el supuesto de la doble imposición. Para tal fin, se debe de asegurar que las obligaciones contraídas se aplican plenamente y de buena fe; que la implementación de procedimientos amistosos fomente la resolución provechosa de todo conflicto de interpretación; y que los contribuyentes tengan acceso a los procedimientos amistosos cuando reúnan las condiciones para ello.

Acción 15: Desarrollar un instrumento multilateral. Con esta acción, y ante la falta de un mecanismo que garantice la rápida implementación de BEPS, se está buscando que instrumento multilateral sea viable técnicamente para implementar las medidas convencionales de BEPS a través de la revisión y rectificación de los actuales convenios fiscales bilaterales, que produciría los mismos efectos que la negociación simultánea de miles de estos convenios.

\section{Inclusión sin distinción al Plan BEPS}

Es importante rescatar que, actualmente más de 60 países en su conjunto han emitido disposiciones en sus legislaciones para atender el paquete de 15 acciones del Pan BEPS, con el fin de combatir la evasión fiscal, mejorar la coherencia de las normas fiscales internacionales y garantizar más transparencia en el ámbito fiscal. Sin embargo, tras la publicación del paquete BEPS, en octubre de 2015, los líderes del G20 tras una evaluación al Plan BEPS solicitaron a la OCDE el desarrollo de un marco más inclusivo, esto es cualquier país interesado, de cualquier jurisdicción, no perteneciente al G20 o con una economía en desarrollo, se le permitiría participar en la implementación de estos lineamientos y medidas relacionadas con los tratados fiscales y BEPS. 
IRRUPCIÓN DE LOS PRECIOS D ETRANSFERENCIA Y LA LUCHA CONTRA EL BEPS

\section{RESULTADOS}

Es importante señalar que, un resultado concluyente consiste en comprender que, la determinación de precios de transferencia no es una ciencia exacta, sin embargo el papel trascendental de la autoridad consistiría en seguir haciendo grandes esfuerzos por legislar con claridad al respecto, partiendo del punto de vista que, por su naturaleza, los análisis de precios de transferencia, más que respuestas definitivas, en general deben ofrecer indicaciones precisas sobre la posición de plena competencia y las estimaciones del precio de plena competencia.

Además de lo anterior, se deberá priorizar el fondo sobre la forma a la hora de la delimitación precisa de la operación, ya que a partir de ella, permitirá seleccionar el método más adecuado para determinar los precios de transferencia.

En particular, el reto para México es precisar una política económica-fiscal más clara y definida para la inversión extranjera en México, ya que el escenario no deseable es que su base fiscal se vea afectada por los precios de transferencia, a su vez que se valoren y consoliden los métodos tradicionales de transacción como la forma preferente de ejecutar el principio de plena competencia, ya que al ser reglas tan generales aun estando en el supuesto del cumplimiento, las serias dudas que surgen en la aplicación de éstas generan temor en las empresas en el sentido de que repercutan directamente en sus resultados fiscales, en su carácter de contribuyentes.

\section{CONCLUSIONES}

Las directrices de Precios de Transferencia de la OCDE están siendo aplicadas por muchos países, impactando contundentemente en la práctica de muchas administraciones tributarias y de empresas multinacionales.

En específico, el plan BEPS de la OCDE/G-20 ofrece un análisis detallado que contempla desde la economía digital y sus modelos de negocio hasta problemas fiscales internacionales que ocupan las agendas políticas de los países, análisis que le permite emitir las medidas y directrices para: Mejorar la coherencia de los estándares impositivos internacionales; evitar la evasión fiscal o la doble imposición; trasladar beneficios hacia ubicaciones donde existe escasa o nula actividad real; garantizar un entorno fiscal de mayor transparencia y; reforzar su focalización en la sustancia económica 
IRRUPCIÓN DE LOS PRECIOS D ETRANSFERENCIA Y LA LUCHA CONTRA EL BEPS

Sin embargo, desde la publicación del plan BEPS, con sus 15 acciones, se han presentado problemas, tales como: Establecer la valoración de intangibles; emitir las recomendaciones que fortalezcan las normas de las Sociedades Extranjeras Controladas; enfrentar a la propiedad intelectual y a los nuevos modelos de negocio; definir el establecimiento permanente y; determinar en la práctica los impuestos que las empresas multinacionales deben pagar según la jurisdicción donde se llevó a cabo la transacción, por mencionar algunos

En consecuencia, la apreciación general para resolver estos problemas que aquejan a las administraciones tributarias y empresas conlleva a reforzar los instrumentos y requerimientos que verdaderamente garanticen los beneficios para los cuales fue creado el plan BEPS y que de hecho, se han realizado tales correcciones para actuar en un plano de igualdad y en un marco de trabajo inclusivo con todos los países interesados con el propósito de implementarlo en la mayor parte del mundo para unificar o estandarizar dichas medidas, así como controlar su impacto en el tiempo.

\section{REFERENCIAS BIBLIOGRÁFICAS}

Alvarado, H. y Vera, R.F. (2016). Nuevo estándar de documentación de precios de transferencia y reporte país por país. Editorial Instituto Mexicano de Contadores Públicos, México.

Cámara Mexicano - Alemana de Comercio e Industria (2018). Plática con las Autoridades Fiscales. Plan BEPS y su efecto en el régimen de precios de transferencia mexicano. https://mexiko.ahk.de/es/eventos/evento-detalles/reunion-del-comite-fiscal/

Comisión de Precios de Transferencia (2011). Precios de Transferencia. Editorial Instituto Mexicano de Contadores Públicos, México.

Congreso de la Unión (2018). Ley del Impuesto sobre la Renta. Última reforma publicada el 30.11.2016. Diario Oficial de la Federación. México.

G20 (2012). Declaración de los Líderes del G20. Los Cabos, México. Recuperado de: www. diplomatie.gouv.fr/fr/IMG/pdf/12-1268-declaration_G20_Los_Cabos_cle82ebc5.pdf.

Hantke, M. (2011). Control de precios de transferencia en la industria de agua potable $y$ alcantarillado. Comisión Económica para América Latina y el Caribe (CEPAL) en conjunto con la Deutsche Gesellschaft für Internationale Zusammenarbeit (GIZ) y financiado por el Ministerio Federal de Cooperación Económica y Desarrollo de Alemania (BMZ). Chile. 


\section{IRRUPCIÓN DE LOS PRECIOS D ETRANSFERENCIA Y LA LUCHA CONTRA EL BEPS}

Martínez, B. (2017). Precios de transferencia, prioridad: OCDE. Recuperado de: https://www.eleconomista.com.mx/economia/Precios-de-transferencia-prioridad-OCDE20170710-0080.html

OCDE. (2018). Base erosion and profit shifting. Recuperado de: http://www.oecd.org/fr/fiscalite/beps/

OCDE. (2017) Transfer Pricing Guidelines for Multinational Enterprises and Tax Administrations. Organisation for Economic Co-operation and Development. Recuperado de https://read.oecdilibrary.org/taxation/oecd-transfer-pricing-guidelines-for-multinational-enterprises-and-taxadministrations-2017/the-arm-s-length-principle_tpg-2017-5-en\#page3.

OCDE. (2015). Proyecto OCDE/G20 sobre la Erosión de la Base Imponible y el Traslado de Beneficios. Resúmenes Informes Finales 2015. Recuperado de: https://www.oecd.org/ctp/beps-resumenes-informes-finales-2015.pdf

OCDE. (2013a). Lucha contra la erosión de la base imponible y el traslado de beneficios. Éditions OCDE, París. Recuperado de: http://dx.doi/org/10.1787/9789264201224-es.

OCDE. (2013b). La relación cooperativa: Un marco de referencia: De la relación cooperativa al cumplimiento cooperativo. Editions OCDE, París. Recuperado de: http://dx.doi/org/10.1787/9789264207547-es.

OCDE. (2013c). Plan de acción contra la erosión de la base imponible y el traslado de beneficios. Recuperado de: http://dx.doi.org/10.1787/9789264207813-es

OCDE (2010) Modelo de Convenio Tributaria sobre la Renta y el Patrimonio, versión abreviada. Recuperado de: http://www.gerens.cl/gerens/ ModeloConvenioTributario.pdf

OCDE (1999) Directrices aplicables en materia de precios de transferencia a empresas multinacionales y administraciones tributarias. En colaboración con la Secretaría de Hacienda y Crédito Público-Secretaría de Administración Tributaria y el Instituto de Estudios Fiscales. México. 